\title{
Quality of life after percutaneous coronary intervention in no-touch saphenous vein grafts is significantly better than in conventional vein grafts
}

\author{
Gabriele Ferrari ${ }^{1}$, Jan Karlsson ${ }^{2}$, Håkan Geijer ${ }^{1}$, Yang Cao ${ }^{3}$, Domingos Souza ${ }^{1}$, and Ninos \\ Samano $^{2}$ \\ ${ }^{1}$ Universitetssjukhuset Orebro Medicinska kliniken \\ ${ }^{2}$ Orebro universitet Medicinska biblioteket \\ ${ }^{3}$ Orebro universitet Centrum for Livsvetenskap
}

November 14, 2021

\begin{abstract}
Objectives: To compare health-related quality of life of patients primarily treated with a no-touch saphenous vein graft with that of patients who received a conventional graft. Methods: The study included all individuals treated with a percutaneous coronary intervention on a saphenous vein graft between January 2006 and June 2020. The RAND-36 health survey was used to assess health-related quality of life. The Mann-Whitney U test was used to test differences in health-related quality of life between the two groups. Effect size was estimated via Cohen's $d$. The average treatment effect between the groups was tested by propensity score matching. Results: Of the 346 patients treated with a percutaneous coronary intervention in a stenosed or occluded saphenous vein graft, 165 responded to RAND-36 (no-touch: $n=48$; conventional: $n=117$ ). Patients with a no-touch graft reported better mean values on seven of the eight health survey domains. Statistically significant differences were observed for four of the domains, all in favour of the no-touch group. The effect size estimates indicated a small difference for five domains, with the largest values $(>0.40)$ seen for the general health and energy/fatigue domains. Propensity score matching confirmed a statistically significant difference for the physical functioning and general health domains. Conclusions: At a mean follow-up of 5.4 years, patients who received a percutaneous coronary intervention in no-touch vein grafts showed significantly better health-related quality of life than those who received a percutaneous coronary intervention in conventional vein grafts.
\end{abstract}

\section{Introduction}

Health-related quality of life (HRQoL) has become an important outcome measure. The majority of medical treatments are now evaluated not only in terms of clinical/biomarker benefits but also in terms of HRQoL improvements. In 2011, Noyez et al. ${ }^{1}$ reviewed the literature regarding HRQoL studies after cardiac surgery. The review showed few HRQoL studies as well as methodological weaknesses such as limited follow-up times and limited sample sizes.

More studies have been published in the last decade, and almost all have used the SF-36 or the RAND-36 to measure HRQoL after cardiac surgery, in particular after coronary artery bypass grafting $(\mathrm{CABG})^{2-11}$. The RAND 36-item health survey $1.0^{12}$ is a public domain and licence-free form equivalent to the SF-36. The scoring for six of the eight subscales is equivalent for the SF-36 and RAND-36, while scoring for the pain and general health scales differs marginally ${ }^{12}$. RAND-36 is a generic measure of HRQoL which has been validated in the general population and for different patient groups. 
To our knowledge, no report published in English has investigated HRQoL in CABG patients who subsequently need a percutaneous coronary intervention (PCI) on a saphenous vein graft (SVG). PCI is an established procedure with excellent results in ischemic heart disease patients, particularly when revascularizing the native coronary arteries ${ }^{13}$. On the other hand, PCI of a degenerated SVG often results in a complex percutaneous intervention and its use is debated ${ }^{14}$. Controversial results with a high rate of major adverse cardiac events (MACE) have been observed in both the short and long term. No results have yet been reported regarding PCI of a saphenous vein harvested with the no-touch $(\mathrm{NT})^{15}$ technique or treated in any other way during the primary CABG operation. The no-touch technique differs from the conventional (C) technique in that it causes less endothelium damage during the harvesting procedure ${ }^{16,17}$, and leads to reduced neo-intimal hyperplasia and subsequent atherosclerosis in the long term ${ }^{18}$. Our group has previously investigated HRQoL in CABG patients who had received a no-touch vein graft ${ }^{11}$, but that study did not compare the no-touch technique with the conventional technique.

The aim of this study was to evaluate HRQoL in individuals who needed a PCI of their SVG after a CABG operation. Our specific aim was to compare HRQoL between patients treated with a no-touch SVG and patients who received a conventional SVG.

\section{Materials and Methods}

\section{Data collection}

The study cohort consisted of all individuals who underwent a CABG operation at our department between January 1992 and May 2020. The present study included all individuals treated with a PCI on the SVG (stenosed or occluded) between 1 January 2006 and 31 May 2020. The SVG was harvested either with the no-touch technique or with the conventional one. Two surgeons reviewed all the surgical reports to check the categorization of cases into the two groups. The PCI was performed in one of two cardiology departments. The sole exclusion criterion was the execution of PCI less than 30 days after the CABG, because this was interpreted as a direct complication of the primary operation due to technical difficulties and not related to the type of vein graft or harvesting technique.

The RAND-36 health survey and information about the study were sent by regular mail to each individual's address (supplementary n.1). In case of non-response, the questionnaire was re-sent at 1-month intervals. All individuals who did not respond the first time were contacted by telephone. Demographic and clinical data were collected from a national quality register, the Swedish cardiological and cardiosurgical intervention register (Swedeheart) ${ }^{19}$, and from the local hospital register. The study has been approved by the Regional Ethics Review Board in Uppsala (DNR: 2015/242).

This was a single-centre study conducted at the centre that invented the no-touch technique and has been using it since 1990.

\section{Patient cohort}

Between 1 January 2006 and 30 June 2020, 346 patients (67 NT, 279 C) who had previously undergone a CABG surgery were treated with a PCI on the SVG. Of these, 16 patients were excluded because the PCI was performed within 30 days of the CABG procedure. A total of 243 patients were alive at the HRQoL follow-up (55 NT and $188 \mathrm{C}$ ) and were asked to participate in the study (Figure 1).

\section{RAND-36 health survey}

The RAND-36 consists of 36 items grouped into eight multi-item scales: physical functioning (PF), rolefunctioning/physical (RP), pain $(\mathrm{P})$, general health $(\mathrm{GH})$, energy/fatigue (EF), social functioning (SF), role-functioning/emotional (RE), and emotional well-being (EW). Scale scores are summed and transformed into scales ranging from 0 (worst possible health state) to 100 (best possible state) ${ }^{12}$.

\section{Statistical methods}

Descriptive statistics were calculated as means, standard deviations (SD), and 95\% confidence intervals 
(CI). Categorical variables were summarized with relative frequency distribution. All continuous data were normally distributed and so summarized with mean and standard deviation (SD). A chi-squared test (or Fisher's exact test if any expected count was lower than 5) was used to compare categorical values between the two groups. An unpaired t-test was performed to compare continuous variables.

Differences in RAND-36 domains between the two treatment groups were tested with the non-parametric Mann-Whitney U test. The magnitudes of group differences were estimated by calculating the effect size (ES; Cohen's $d$ ). ES makes it possible to interpret the importance of a group difference and facilitates comparison across different measures. ES was calculated as the mean difference between groups divided by the pooled $\mathrm{SD}^{20}$, and was judged according to the standard criteria proposed by Cohen: trivial $(0.0$ to $<0.2)$, small $(0.2$ to $<0.5)$, medium $(0.5$ to $<0.8)$, and large $([?] 0.8)$.

In addition, propensity score matching (PSM) was used to estimate the average treatment effects between the two groups, using 1:1 nearest neighbour matching based on the propensity scores. The matched sample size was 96 (48:48). PSM was used as a sensitivity analysis to assess the robustness of the primary analysis results. Propensity scores of patients treated with no-touch or conventional SVG were estimated using a logistic regression model with age, sex, smoking, hypertension, diabetes mellitus, and creatinine level as predictors $^{21}$.

The statistical analyses were performed in version 27.0 of SPSS (IBM, Armonk, NY, USA) and version 16.1 of Stata (StataCorp, College Station, TX, USA).

\section{Results}

The study included 243 individuals treated with a PCI in a stenosed or occluded SVG and alive at survey time. A total of 165 (67.9\%) individuals responded to the RAND-36 health survey, 48 (87.3\%) in the no-touch group and $117(62.2 \%)$ in the conventional group (Figure 1).

\section{Demographic and clinical characteristics}

The demographic and surgical characteristics of the two treatment groups are presented in Tables 1 and 2. The demographic characteristics of the two groups were quite similar, with comparable mean age at time of CABG $(57.1 \pm 8.6$ years vs. $57.2 \pm 8.1$ years; $\mathrm{p}=0.93)$, at time of PCI $(\mathrm{p}=0.72)$, and at time of HRQoL follow-up $(73.4 \pm 8.8$ years vs. $75.4 \pm 7.2$ years; $\mathrm{p}=0.12)$. Most of the patients were male (around $80 \%$ ). Risk factors were equally distributed in the two groups, and all comparisons of risk factors between the groups were non-significant. Regarding the characteristics of the CABG and the PCI procedures (Table 2 ), the two groups showed comparable data in terms of number of anastomoses performed at the CABG, number of successful PCI procedures, frequency of distal embolic protection device usage, and number of thrombectomies performed. The only significant difference was the use of dual antiplatelet therapy at baseline (NT: $54.2 \%$ vs. C: $72.6 \%$; p=0.02). However, after the PCI, according to local protocol, the patients received dual antiplatelet therapy for at least 1 year if no contraindication was present.

Analysis of the number of cardiovascular events at 1 year (Table 3) and at long-term follow-up (Table 4) confirmed the comparability of the two groups. At 1 year after the PCI, no differences in term of MACE, in-stent restenosis, or re-hospitalization were reported (Table 3). At the time of HRQoL follow-up (Table 4), more events were reported in the conventional group, but the between-group differences were not statistically significant except for the frequency of in-stent restenosis (NT: $6.25 \%$ vs. C: $22.2 \%$; $=0.01$ ).

\section{RAND-36 health survey}

RAND-36 health profiles divided by type of vein graft are presented in Table 5. The patients treated with a notouch vein graft reported higher mean values in seven of the eight health domains, indicating better HRQoL. Differences between the two groups were statistically significant $(\mathrm{p}<0.05)$ in four of the eight domains, all in favour of patients with the no-touch vein graft treatment $(\mathrm{p}=0.028$ for physical functioning, $\mathrm{p}=0.022$ for general health, $\mathrm{p}=0.010$ for energy/fatigue, and $\mathrm{p}=0.026$ for emotional well-being). In terms of ES, the between-group differences were trivial $(\mathrm{ES}<0.20)$ for the role functioning/physical, social functioning, and 
role functioning/emotional domains, and small $(0.20$ [?] ES $<0.50)$ for the other five domains (Table 5). The largest ESs $(>0.40)$ were noted for the general health and energy/fatigue domains.

PSM analysis (Table 6) confirmed a statistically significant difference between the two groups for the physical functioning $(\mathrm{p}=0.041)$ and general health domains $(\mathrm{p}=0.002)$. The difference for the energy/fatigue domain showed a borderline trend towards statistical significance $(\mathrm{p}=0.056)$.

\section{Discussion}

Percutaneous coronary intervention on SVGs has been in continuous evolution over recent decades. It currently represents approximately $6 \%$ of all percutaneous coronary procedures in the US ${ }^{22}$. Patients undergoing PCI in SVGs have more early and late adverse cardiac events ${ }^{23}$, which might predispose them to a deterioration of their HRQoL and increase the burden for healthcare. This is the first study to show a better HRQoL after PCI in no-touch versus conventional vein grafts.

Few studies have evaluated HRQoL outcomes after PCI in a vein graft. Our group ${ }^{11}$ used the EQ-5D-3L questionnaire to examine HRQoL in individuals after $\mathrm{CABG}$, and concluded that the graft patency was associated with better HRQoL. However, the HRQoL outcome of the no-touch or conventional vein graft technique was not evaluated separately.

The present study evaluated HRQoL using the RAND-36 health survey in patients treated with no-touch or conventional vein grafts. Our primary analysis showed significant differences between the two treatment groups in four of the eight RAND-36 domains (PF, GH, EF, EW), indicating better HRQoL in the no-touch group at a mean of $5.4+-3.6$ years after PCI. The effect size estimates (Cohen's $d$ ) indicated better HRQoL in the no-touch group in five domains (PF, P, GH, EF, EW). Effect sizes were small, but the difference in scale scores on the physical functioning and energy/fatigue scales was approximately 10 points, which has been referred to as a medium group difference ${ }^{24}$. The energy/fatigue domain showed the largest difference $(\mathrm{p}=0.010 ; \mathrm{ES}=0.43)$. One possible explanation for these results could be the higher in-stent restenosis rate after PCI in the conventional group, and its consequences in terms of quality of life. Further clarifications in terms of clinical outcomes are under investigation with a larger patient cohort (ClinicalTrials.gov no. NCT03999398).

Few studies have estimated the minimal clinically important difference (MCID) for the RAND-36/SF36 scales in cardiopathic populations. Bjorner et al. evaluated MCID for energy/fatigue in individuals with chronic conditions including congestive heart failure, and recommended a MCID of 5-10 points ${ }^{25}$. In the present study, the difference between the no-touch and the conventional group was 9.6 points for energy/fatigue, indicating a clinically important difference.

The patients who received a no-touch vein graft estimated their physical health (PF, RP, P, GH) more positively than the conventional group, although the differences in RP and $\mathrm{P}$ were not significant. The positive effect on physical health in the no-touch group was further confirmed by the PSM analysis, showing statistically significant differences in two domains (PF, GH). Comparison of the average treatment effects according to the PSM analysis (Table 6) and the differences between the treatment groups according to the primary analysis (Table 5) showed that the results were roughly equal. The general similarity between the primary analysis and the PSM indicates that no predictor variable behaved as a confounder in the analysis.

The confirmed positive effect on the physical health components supports the clinical relevance of the HRQoL difference between the two techniques; since, the scales that primarily measure physical health are associated particularly with the health condition in cardiac and cardio-operated patients ${ }^{26}$.

Hokkanen et al. ${ }^{6}$ used RAND-36 to examine both short-term (1 year) and long-term (12 years) changes in HRQoL in patients treated with CABG. Their 1-year results ${ }^{27}$ demonstrated that all RAND-36 domains improved significantly; however, this improvement was significant only among patients under 75 years. At the 12-year follow-up, significant improvements were observed in all RAND-36 domains except general health. Moreover, patients younger than 65 years at baseline maintained their physical health status after 12 years, whereas the older patients reported a pronounced decrease in both the physical and mental component 
summary scores. The present study was similar in terms of follow-up time, although it was not prospective and did not analyse changes over time. However, an analysis of our results for patients under 65 years showed RAND-36 scores comparable to those reported by Hokkanen et al., particularly in the no-touch group. It is noteworthy that the general health domain did not improve in the earlier study, with a mean value of 54.2 at baseline and 54.5 at 12 years ${ }^{6}$. Our study found a significant difference in general health between the no-touch and conventional group, with a mean value of 61.0 in the no-touch group; the effect size was in the upper range of a small difference $(\mathrm{ES}=0.41)$. This result can be explained by the already-known fact that no-touch vein graft patients tend to have reduced atherosclerosis over time and lower rates of adverse cardiac events $^{28}$, with an expected positive impact on general health.

Few studies have evaluated HRQoL after PCI using the SF-36 or the RAND-36 ${ }^{29,} 30$. In 2008, Gunal et al. ${ }^{30}$ reported SF-36 results for octogenarians treated with PCI that partially differ from our results. Their study group showed a markedly lower mean value on the physical functioning scale $(41+-28)$ than we found in our study, which may be explained by the demographic characteristics of the study population (patients over 80 years). However, scores on the pain, role-emotional, and emotional well-being scales were comparable with the results for the conventional group in the present study. Cohen et al. ${ }^{29}$ investigated HRQoL using the SF36 after either a PCI or a CABG at 1 month, 6 months, and 1 year after the intervention, finding no change in HRQoL at the 1-year follow-up. Their SF-36 results after 1 year were equivalent to those observed in the no-touch group in our study, except for physical functioning, which was better in the study by Cohen et al., and mental health/emotional well-being, which was better in our study. This comparison between the PCI of a native vessel ${ }^{31}$ and the PCI of a no-touch vein graft suggests the hypothesis that no-touch SVGs are as suitable for PCI as a native coronary artery. A better HRQoL score thus correlates with the reduced intimal damage and subsequent atherosclerosis in the long term during the harvesting of the no-touch veins ${ }^{18}$.

The main limitation of the present study is the small number of individuals in the no-touch group $(\mathrm{n}=48)$, despite the high response rate $(87.3 \%)$. However, the power was sufficient to detect significant differences between the two study groups on four of the eight RAND-36 scales. We also calculated effect sizes, which are independent of sample size, to estimate the magnitude of group differences. A second limitation is the retrospective study design, which means that HRQoL data before the operation were not available. Another possible limitation is the difference between the study groups in terms of follow-up time. Individuals treated with no-touch had shorter follow-up time than the conventionally treated patients (4.4 vs. 5.9 years; see Table 1). According to Hokkanen et al. ${ }^{6}$, a main predictor of HRQoL after CABG is the initial age at CABG, with declining RAND-36 scores at the 12-year follow-up in patients who were older than 65 years at the time of the CABG. Our study groups showed comparable age at time of CABG, PCI, and HRQoL follow-up, indicating that age was not a confounding factor in the comparison between the two groups.

\section{Conclusion}

At a mean follow-up of 5.4 years, patients who received PCI in no-touch SVGs showed significantly better HRQoL than those who received PCI in conventional vein grafts.

Conflict of Interest statement: None declared.

Funding Statement: This work was supported by Region Orebro County through the regional research board (grant OLL-935188, 18 January 2020).

\section{Clinical registration number}

This study was registered with ClinicalTrials.gov (no. NCT03999398, 25 June 2019), and with the Research and Development registry in Sweden (project no: OLL-242381, 17 October 2017).

\section{Author contribution statement}

Gabriele Ferrari, Hakan Geijer, Ninos Samano, and Domingos Souza originated the concept of this study. Gabriele Ferrari, Hakan Geijer, Jan Karlsson, and Ninos Samano designed the study. Gabriele Ferrari and Hakan Geijer collected the patient data. Gabriele Ferrari, Jan Karlsson, and Yang Cao performed the 
statistical analyses. Gabriele Ferrari and Jan Karlsson wrote the first draft of the manuscript. All authors reviewed the manuscript, contributed significantly to its critical review, and approved the final version.

\section{Declaration of Helsinki}

The study was performed in accordance with the Declaration of Helsinki.

\section{References}

1. Noyez L, de Jager MJ, Markou AL. Quality of life after cardiac surgery: underresearched research. Interact Cardiovasc Thorac Surg 2011;13(5):511-4.

2. Coelho P, Miranda L, Barros PMP, Fragata JIG. Quality of life after elective cardiac surgery in elderly patients. Interact Cardiovasc Thorac Surg 2019;28(2):199-205.

3. Fox NL, Hoogwerf BJ, Czajkowski S, Lindquist R, Dupuis G, Herd JA, Campeau L, Hickey A, Barton FB, Terrin ML. Quality of life after coronary artery bypass graft: results from the POST CABG Trial. Chest 2004;126(2):487-95.

4. Gjeilo KH, Stenseth R, Wahba A, Lydersen S, Klepstad P. Long-term health-related quality of life and survival after cardiac surgery: A prospective study. J Thorac Cardiovasc Surg 2018;156(6):2183-90.e2.

5. Grand N, Bouchet JB, Zufferey P, Beraud AM, Awad S, Sandri F, Campisi S, Fuzellier JF, Molliex S, Vola M, Morel J. Quality of Life After Cardiac Operations Based on the Minimal Clinically Important Difference Concept. Ann Thorac Surg 2018;106(2):548-54.

6. Hokkanen M, Jarvinen O, Huhtala H, Tarkka MR. A 12-year follow-up on the changes in health-related quality of life after coronary artery bypass graft surgery. Eur J Cardiothorac Surg 2014;45(2):329-34.

7. Jarvinen O, Hokkanen M, Huhtala H. Diabetics have Inferior Long-Term Survival and Quality of Life after CABG. Int J Angiol 2019;28(1):50-6.

8. Pačarić S, Turk T, Erić I, Orkić Ž, Petek Erić A, Milostić-Srb A, Farčić N, Barać I, Nemčić A. Assessment of the Quality of Life in Patients before and after Coronary Artery Bypass Grafting (CABG): A Prospective Study. Int J Environ Res Public Health 2020;17(4).

9. Peric V, Stolic R, Jovanovic A, Grbic R, Lazic B, Sovtic S, Borzanovic M. Predictors of Quality of Life Improvement after 2 Years of Coronary Artery Bypass Surgery. Ann Thorac Cardiovasc Surg 2017;23(5):2338.

10. Perrotti A, Ecarnot F, Monaco F, Dorigo E, Monteleone P, Besch G, Chocron S. Quality of life 10 years after cardiac surgery in adults: a long-term follow-up study. Health Qual Life Outcomes 2019;17(1):88.

11. Samano N, Bodin L, Karlsson J, Geijer H, Arbeus M, Souza D. Graft patency is associated with higher health-related quality of life after coronary artery bypass surgery. Interact Cardiovasc Thorac Surg 2017;24(3):388-94.

12. Hays RD, Sherbourne CD, Mazel RM. The RAND 36-Item Health Survey 1.0. Health Econ 1993;2(3):21727.

13. Brilakis ES, O'Donnell CI, Penny W, Armstrong EJ, Tsai T, Maddox TM, Plomondon ME, Banerjee S, Rao SV, Garcia S, Nallamothu B, Shunk KA, Mavromatis K, Grunwald GK, Bhatt DL. Percutaneous Coronary Intervention in Native Coronary Arteries Versus Bypass Grafts in Patients With Prior Coronary Artery Bypass Graft Surgery: Insights From the Veterans Affairs Clinical Assessment, Reporting, and Tracking Program. JACC Cardiovasc Interv 2016;9(9):884-93.

14. Ferrari G, Geijer H, Cao Y, Souza D, Samano N. Percutaneous coronary intervention in saphenous vein grafts after coronary artery bypass grafting: a systematic review and meta-analysis. Scand Cardiovasc J 2021:1-9. 
15. Souza D. A new no-touch preparation technique. Technical notes. Scand J Thorac Cardiovasc Surg 1996;30(1):41-4.

16. Ramos De Souza D, Dashwood MR, Samano N. Saphenous vein graft harvesting and patency: No-touch harvesting is the answer. J Thorac Cardiovasc Surg 2017;154(4):1300-1.

17. Samano N, Dashwood M, Souza D. No-touch vein grafts and the destiny of venous revascularization in coronary artery bypass grafting-a 25(th) anniversary perspective. Ann Cardiothorac Surg 2018;7(5):681-5.

18. Johansson BL, Souza DS, Bodin L, Filbey D, Loesch A, Geijer H, Bojö L. Slower progression of atherosclerosis in vein grafts harvested with 'no touch' technique compared with conventional harvesting technique in coronary artery bypass grafting: an angiographic and intravascular ultrasound study. Eur J Cardiothorac Surg 2010;38(4):414-9.

19. Swedeheart. 2020 [cited 202030 November 2020]. Available from:https://www.ucr.uu.se/swedeheart/.

20. Fayers P, and David Machin. Quality of Life : The Assessment, Analysis and Reporting of PatientReported Outcomes; 2015.

21. Deb S, Austin PC, Tu JV, Ko DT, Mazer CD, Kiss A, Fremes SE. A Review of Propensity-Score Methods and Their Use in Cardiovascular Research. Can J Cardiol 2016;32(2):259-65.

22. Brilakis ES, Rao SV, Banerjee S, Goldman S, Shunk KA, Holmes DR, Jr., Honeycutt E, Roe MT. Percutaneous coronary intervention in native arteries versus bypass grafts in prior coronary artery bypass grafting patients: a report from the National Cardiovascular Data Registry. JACC Cardiovasc Interv 2011;4(8):844-50.

23. Lichtenwalter C, de Lemos JA, Roesle M, Obel O, Holper EM, Haagen D, Saeed B, Iturbe JM, Shunk K, Bissett JK, Sachdeva R, Voudris VV, Karyofillis P, Kar B, Rossen J, Fasseas P, Berger P, Banerjee S, Brilakis ES. Clinical presentation and angiographic characteristics of saphenous vein graft failure after stenting: insights from the SOS (stenting of saphenous vein grafts) trial. JACC Cardiovasc Interv 2009;2(9):855-60.

24. Ware J, Snoww K, Ma K, Bg G. SF36 Health Survey: Manual and Interpretation Guide. Lincoln, RI: Quality Metric, Inc, 1993 1993;30.

25. Bjorner JB, Wallenstein GV, Martin MC, Lin P, Blaisdell-Gross B, Tak Piech C, Mody SH. Interpreting score differences in the SF-36 Vitality scale: using clinical conditions and functional outcomes to define the minimally important difference. Curr Med Res Opin 2007;23(4):731-9.

26. Lahoud R, Chongthammakun V, Wu Y, Hawwa N, Brennan DM, Cho L. Comparing SF-36ß) scores versus biomarkers to predict mortality in primary cardiac prevention patients. Eur J Intern Med 2017;46:4755.

27. Järvinen O, Saarinen T, Julkunen J, Huhtala H, Tarkka MR. Changes in health-related quality of life and functional capacity following coronary artery bypass graft surgery. Eur J Cardiothorac Surg 2003;24(5):750-6.

28. Samano N, Geijer H, Liden M, Fremes S, Bodin L, Souza D. The no-touch saphenous vein for coronary artery bypass grafting maintains a patency, after 16 years, comparable to the left internal thoracic artery: A randomized trial. J Thorac Cardiovasc Surg 2015;150(4):880-8.

29. Cohen DJ, Van Hout B, Serruys PW, Mohr FW, Macaya C, den Heijer P, Vrakking MM, Wang K, Mahoney EM, Audi S, Leadley K, Dawkins KD, Kappetein AP. Quality of life after PCI with drug-eluting stents or coronary-artery bypass surgery. N Engl J Med 2011;364(11):1016-26.

30. Günal A, Aengevaeren WR, Gehlmann HR, Luijten JE, Bos JS, Verheugt FW. Outcome and quality of life one year after percutaneous coronary interventions in octogenarians. Neth Heart J 2008;16(4):117-22.

\section{Figure legends}

Figure 1: PRISMA flowchart of the individuals included in the study 
Table 1: Demographic characteristics

Table 2: Surgical and percutaneous coronary intervention (PCI) characteristics

Table 3: Cardiac events at 1 year

Table 4: Cardiac events at the health-related quality of life follow-up

Table 5: Results of the RAND-36 health survey divided by vein graft type

Table 6: Treatment effects on the RAND-36 domains of no-touch versus conventional vein graft (propensity score matching)

Figures

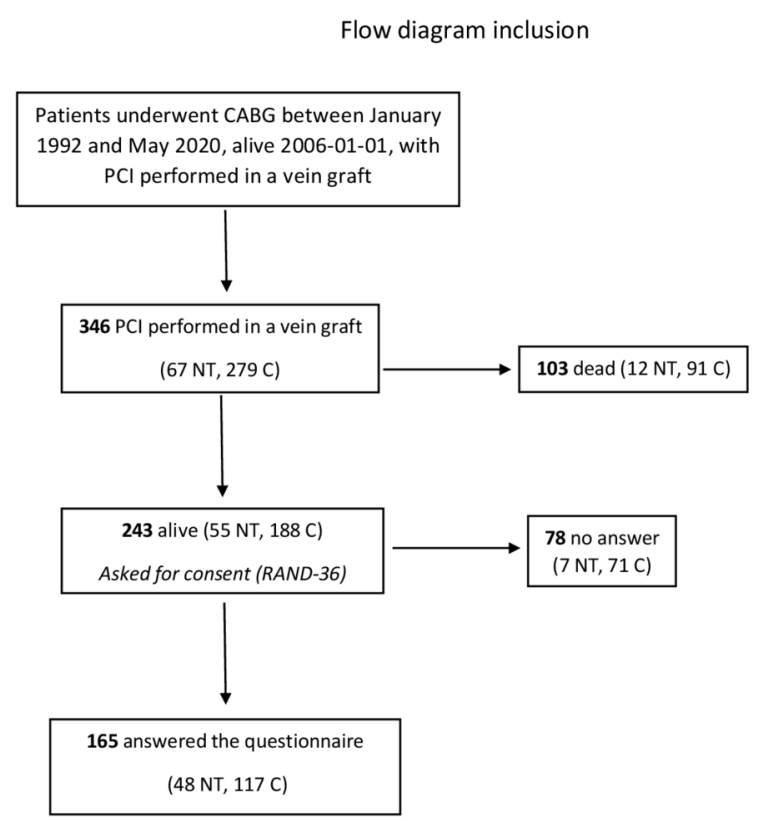


Figure 1: PRISMA flowchart of the individuals included in the study. C: conventional graft; CABG: coronary artery bypass grafting; NT: no-touch graft; PCI: percutaneous coronary intervention.

Tables

No. of patients

Age at CABG

Age at PCI

Age at survey answer

Time between CABG and PCI

Time between PCI and survey

Male gender

Smoking (past and present)

Hypertension

Diabetes mellitus

Creatinine level

\begin{tabular}{llll}
$\begin{array}{l}\text { No-touch } \\
48\end{array}$ & Conventional & Conventional & p-value \\
$57.1 \pm 8.6$ & $57.2 \pm 8.1$ & 0.935 & 0.935 \\
$70.1 \pm 9.1$ & $71.2 \pm 8.1$ & 0.725 & 0.725 \\
$73.4 \pm 8.8$ & $75.4 \pm 7.21$ & 0.124 & 0.124 \\
$13.6 \pm 5.9$ & $14.1 \pm 4.8$ & 0.534 & 0.534 \\
$4.4 \pm 3.9$ & $5.9 \pm 3.5$ & 0.021 & 0.021 \\
$38(79.2 \%)$ & $104(88.9 \%)$ & 0.102 & 0.102 \\
$25(52.1 \%)$ & $74(63.2 \%)$ & 0.276 & 0.276 \\
$43(89.6 \%)$ & $98(83.7 \%)$ & 0.622 & 0.622 \\
$16(33.3 \%)$ & $37(31.6 \%)$ & 0.831 & 0.831 \\
$83.8 \pm 25.0$ & $90.4 \pm 28.3$ & 0.185 & 0.185 \\
\hline
\end{tabular}

\section{Table 1: Demographic characteristics}

Values are presented as mean \pm standard deviation or $\mathrm{n}(\%)$. Ages and times are given in years, and creatinine is given in micromole/L. Smoking was divided into three categories in the analysis: never smokers, past smokers (more than 1 month ago), present smokers.

Table 2: Surgical and percutaneous coronary intervention (PCI) characteristics

\begin{tabular}{llll}
\hline & No-touch & Conventional & p-value \\
No. of patients & 48 & 117 & \\
Number of distal anastomoses & $3.7 \pm 1.1$ & $3.6 \pm 0.9$ & 0.585 \\
PCI success (restenosis <20\%) & $42(87.5 \%)$ & $104(88.9 \%)$ & 0.800 \\
Not possible to perform PCI & $3(6.3 \%)$ & $10(8.5 \%)$ & 0.758 \\
Distal embolic protection device & $1(2.1 \%)$ & $5(4.3 \%)$ & 0.673 \\
Thrombectomy performed & $6(12.5 \%)$ & $9(7.7 \%)$ & 0.374 \\
Dual antiplatelet therapy & $26(54.2 \%)$ & $85(72.6 \%)$ & 0.022 \\
\hline
\end{tabular}

Values are presented as mean \pm standard deviation or $\mathrm{n}(\%)$.

Table 3: Cardiac events at 1 year

\begin{tabular}{llll}
\hline $\begin{array}{l}\text { No. of patients } \\
\begin{array}{l}\text { Re-angiography during } \\
\text { first year }\end{array}\end{array}$ & $\begin{array}{l}\text { No-touch } \\
\text { None }\end{array}$ & $\begin{array}{l}\text { Conventional } \\
117\end{array}$ & p-value \\
1 & $43(89.6 \%)$ & $102(87.2 \%)$ & 0.706 \\
2 & $5(10.4 \%)$ & $13(11.1 \%)$ & \\
$\begin{array}{l}\text { Cardiac hospitalization } \\
\text { during first year }\end{array}$ & $0(0 \%)$ & $2(1.7 \%)$ & \\
$\begin{array}{l}\text { In-stent restenosis } \\
\text { during first year }\end{array}$ & $0(0 \%)$ & $23(19.7 \%)$ & 0.083 \\
& & $1(0.8 \%)$ & 1.000
\end{tabular}


Values are presented as $\mathrm{n}(\%)$.

Table 4: Cardiac events at health-related quality of life follow-up

\begin{tabular}{|c|c|c|c|}
\hline & No-touch & Conventional & p-value \\
\hline No. of patients & 48 & 117 & \\
\hline $\begin{array}{l}\text { Re-angiography at } \\
\text { follow-up }\end{array}$ & & & 0.091 \\
\hline None & $35(72.9 \%)$ & $72(61.5 \%)$ & \\
\hline 1 & $11(22.9 \%)$ & $26(22.2 \%)$ & \\
\hline 2 & $1(2.1 \%)$ & $9(7.7 \%)$ & \\
\hline 3 & $1(2.1 \%)$ & $10(8.5 \%)$ & \\
\hline $\begin{array}{l}\text { In-stent restenosis at } \\
\text { follow-up }\end{array}$ & $3(6.3 \%)$ & $26(22.2 \%)$ & 0.015 \\
\hline $\begin{array}{l}\text { Major adverse cardiac } \\
\text { event at follow-up }\end{array}$ & $13(27.1 \%)$ & $46(39.3 \%)$ & 0.072 \\
\hline
\end{tabular}

Table 5: Results of the RAND-36 health survey divided by vein graft type

\begin{tabular}{|c|c|c|c|c|c|c|c|c|c|}
\hline \multirow[t]{2}{*}{$\begin{array}{l}\text { RAND- } \\
36 \\
\text { domains }\end{array}$} & $\begin{array}{l}\text { No- } \\
\text { touch } \\
(n=48)\end{array}$ & $\begin{array}{l}\text { No- } \\
\text { touch } \\
(n=48)\end{array}$ & $\begin{array}{l}\text { No- } \\
\text { touch } \\
(n=48)\end{array}$ & $\begin{array}{l}\text { Conventio } \\
(\mathrm{n}=117)\end{array}$ & $\begin{array}{c}\text { nabnventio } \\
(\mathrm{n}=117)\end{array}$ & $\begin{array}{l}(\mathrm{n}=117) \\
(\mathrm{n}=1 \mathrm{~b}\end{array}$ & $\begin{array}{l}\text { nalfference } \\
\text { between } \\
\text { groups }\end{array}$ & $\begin{array}{l}\text { Difference } \\
\text { between } \\
\text { groups }\end{array}$ & p-value \\
\hline & Mean & SD & $\begin{array}{l}95 \% \\
\text { CI }\end{array}$ & Mean & SD & $\begin{array}{l}95 \% \\
\text { CI }\end{array}$ & Mean & $\begin{array}{l}95 \% \\
\text { CI }\end{array}$ & \\
\hline $\mathrm{PF}$ & 68.6 & 26.7 & $\begin{array}{l}62.5- \\
77.9\end{array}$ & 58.4 & 27.6 & $\begin{array}{l}53.8- \\
63.9\end{array}$ & 10.3 & $\begin{array}{l}1.0- \\
19.5\end{array}$ & 0.028 \\
\hline RP & 47.4 & 42.6 & $\begin{array}{l}36.2- \\
62.6\end{array}$ & 40.3 & 40.0 & $\begin{array}{l}32.6- \\
47.6\end{array}$ & 7.0 & $\begin{array}{l}-6.8- \\
20.1\end{array}$ & 0.352 \\
\hline $\mathrm{P}$ & 71.8 & 25.5 & $\begin{array}{l}64.2- \\
79.4\end{array}$ & 65.0 & 29.3 & $\begin{array}{l}59.9- \\
70.7\end{array}$ & 6.8 & $\begin{array}{l}-2.9- \\
16.5\end{array}$ & 0.171 \\
\hline GH & 61.0 & 19.3 & $\begin{array}{l}55.2- \\
66.7\end{array}$ & 52.6 & 21.0 & $\begin{array}{l}49.0- \\
56.8\end{array}$ & 8.3 & $\begin{array}{l}1.3- \\
15.4\end{array}$ & 0.022 \\
\hline $\mathrm{EF}$ & 65.4 & 21.8 & $\begin{array}{l}58.9- \\
71.9\end{array}$ & 55.8 & 22.7 & $\begin{array}{l}51.2- \\
59.9\end{array}$ & 9.6 & $\begin{array}{l}1.9- \\
17.4\end{array}$ & 0.010 \\
\hline SF & 77.2 & 27.5 & $\begin{array}{l}69.1- \\
85.4\end{array}$ & 72.9 & 27.6 & $\begin{array}{l}68.2- \\
78.4\end{array}$ & 4.3 & $\begin{array}{l}-5.2- \\
13.8\end{array}$ & 0.243 \\
\hline $\mathrm{RE}$ & 70.1 & 40.2 & $\begin{array}{l}60.8- \\
84.1\end{array}$ & 69.7 & 39.5 & $\begin{array}{l}62.9- \\
77.7\end{array}$ & 0.4 & $\begin{array}{l}-13.1- \\
13.9\end{array}$ & 0.851 \\
\hline EW & 81.0 & 21.0 & $\begin{array}{l}74.8- \\
87.3\end{array}$ & 74.9 & 20.4 & $\begin{array}{l}71.3- \\
79.1\end{array}$ & 6.1 & $\begin{array}{l}-0.9- \\
13.2\end{array}$ & 0.026 \\
\hline
\end{tabular}

Effect size was calculated according to Cohen's $d$ and categorized as trivial $(0.0$ to $<0.2)$, small $(0.2$ to $<0.5)$, medium $(0.5$ to $<0.8)$, or large ([?]0.8). PF: physical functioning; RP: role functioning/physical; P: pain; GH: general health; EF: energy/fatigue; SF: social functioning; RE: role functioning/emotional; EW: emotional well-being. Significant p-values are shown in bold. Effect sizes categorized as at least "small" are underlined. 
Table 6: Treatment effects on the RAND-36 domains of no-touch versus conventional vein graft (propensity score matching)

\begin{tabular}{lll}
\hline RAND-36 domains & Average treatment effect $(\mathbf{9 5 \%}$ CI) & p-value \\
Physical functioning & $\mathbf{9 . 3 1}(\mathbf{0 . 3 8}-\mathbf{1 8 . 2 4})$ & $\mathbf{0 . 0 4 1}$ \\
Role functioning/physical & $6.03(-8.27-20.33)$ & 0.409 \\
Pain & $6.83(-2.92-16.57)$ & 0.170 \\
General health & $\mathbf{1 0 . 3 1 ( 3 . 8 5 - 1 6 . 7 6 )}$ & $\mathbf{0 . 0 0 2}$ \\
Energy/fatigue & $8.72(-0.24-17.68)$ & 0.056 \\
Social functioning & $3.86(-5.73-13.45)$ & 0.430 \\
Role functioning/emotional & $-0.82(-15.13-13.49)$ & 0.911 \\
Emotional well-being & $4.62(-5.78-15.02)$ & 0.384 \\
\hline
\end{tabular}

\section{Hosted file}

figure 1.docx available at https://authorea.com/users/446023/articles/545371-quality-oflife-after-percutaneous-coronary-intervention-in-no-touch-saphenous-vein-grafts-issignificantly-better-than-in-conventional-vein-grafts

\section{Hosted file}

Tables.docx available at https://authorea.com/users/446023/articles/545371-quality-oflife-after-percutaneous-coronary-intervention-in-no-touch-saphenous-vein-grafts-issignificantly-better-than-in-conventional-vein-grafts 